\title{
MicroRNA-155 promotes apoptosis of colonic smooth muscle cells and aggravates colonic dysmotility by targeting IGF-1
}

\author{
XIAOXUE SHEN, ZHIBIN ZHAO and BIN YANG \\ Department of Gastroenterology, Jiangsu Taizhou People's Hospital, Taizhou, Jiangsu 225300, P.R. China
}

Received October 8, 2018; Accepted June 13, 2019

DOI: $10.3892 /$ etm.2020.8485

\begin{abstract}
Colonic dysmotility as a complication of diabetes affects public health; however, the underlying molecular mechanisms have remained elusive. Insulin-like growth factor-1 (IGF-1) was previously demonstrated to prevent apoptosis of colonic smooth muscle cells (SMCs) and alleviate colonic dysmotility in diabetic rats. However, the regulatory mechanisms upstream of IGF-1 in colonic dysmotility have remained to be determined. The present study reports on microRNA-155 (miR-155), initially identified using bioinformatics, as a direct upstream regulator of IGF-1. In colonic SMCs, miR-155 negatively regulated IGF-1 expression at the post-transcriptional level, as identified through ectopic overexpression and knockdown experiments. A luciferase reporter assay further demonstrated that miR-155 inhibits IGF-1 through binding to its 3'-untranslated region. Furthermore, overexpression of miR-155 led to increased apoptosis of colonic SMCs and a decrease in the thickness of colonic smooth muscle tissues of diabetic mice, indicating miR-155 aggravates colonic dysmotility. By contrast, knockdown of miR-155 induced the opposite effect. Overall, the results of the present study suggest a role of miR-155 in colonic dysmotility, thereby providing a novel therapeutic target.
\end{abstract}

\section{Introduction}

Diabetes mellitus is a chronic disease caused by metabolic disorders and is frequently accompanied by numerous complications $(1,2)$; it has become a serious threat to public health. One of the common complications is gastrointestinal dysfunction, which usually presents as gastric/colonic dysmotility (3-5). However, the underlying molecular mechanisms, which may provide novel and practical therapeutic strategies, remain largely elusive. It is known that gastrointestinal motility

Correspondence to: Dr Bin Yang, Department of Gastroenterology, Jiangsu Taizhou People's Hospital, 366 Taihu Road, High-tech Zone, Taizhou, Jiangsu 225300, P.R. China

E-mail: xjshzdxtz@sina.com

Key words: microRNA-155, insulin-like growth factor-1, apoptosis, colonic dysmotility results from the coordinated contractions of smooth muscle cells (SMCs) (6). A study by Sun et al (7) has indicated that insulin-like growth factor-1 (IGF-1) may prevent apoptosis of colonic SMCs and alleviate colonic dysmotility in diabetic rats. This previous study suggests a key role of IGF-1 in colonic dysmotility. However, the upstream regulatory mechanisms of IGF-1 in colonic SMCs and colonic dysmotility remain to be explored.

microRNAs (miRNAs/miRs) are a group of endogenous, small non-coding RNAs, which usually have a length of $\sim 22$ nucleotides and regulate gene expression at the post-transcriptional level $(8,9)$. In general, miRNAs function by binding to the 3'-untranslated regions (3'-UTRs) of target mRNAs, leading to translational repression or mRNA degradation. Of note, miRNAs regulate $>60 \%$ of mammalian protein-coding genes (10-12). Therefore, miRNAs are involved in almost all cellular processes, including proliferation, differentiation and apoptosis (9). Furthermore, miRNAs have pivotal roles in physiology and pathology (13-16). miR-155 is one of the miRNAs that is known to regulate physiological and pathological processes. For instance, miR-155 has been identified as a tumor-suppressive miRNA in colon cancer through targeting collagen triple helix repeat containing 1 or forkhead box O3 $(17,18)$. In addition, miR-155 is able to mediate endothelial progenitor cell dysfunction caused by high glucose through targeting patched-1 (19) and has been reported to regulate the inflammatory response in the colonic mucosa (20). However, the role of miR-155 in colonic SMCs and colonic dysmotility has remained elusive.

In the present study, miR-155 was identified to directly target IGF-1 to promote apoptosis of colonic SMCs. Furthermore, miR-155 was identified to aggravate colonic dysmotility in diabetic mice through targeting IGF-1.

\section{Materials and methods}

Cells. Mouse colonic SMCs were purchased from Rochen Pharma Co., Ltd. (cat. no. RC-RM-0052) and cultured in Dulbecco's modified Eagle's medium (Thermo Fisher Scientific, Inc.) supplemented with $15 \%$ fetal bovine serum (Thermo Fisher Scientific, Inc.) at $37^{\circ} \mathrm{C}$ with $5 \% \mathrm{CO}_{2}$.

Protein extraction and western blot analysis. The colonic tissue samples were frozen in liquid nitrogen, ground into powder, lysed using radioimmunoprecipitation assay lysis 
buffer (Thermo Fisher Scientific, Inc.) containing the protease inhibitor cocktail (Thermo Fisher Scientific, Inc.) and incubated on ice for $30 \mathrm{~min}$. Tissue homogenates and cell lysates were then centrifuged for $10 \mathrm{~min}$ at $12,000 \mathrm{x} \mathrm{g}$ and $4^{\circ} \mathrm{C}$ and the protein concentration of the supernatant was determined with the Pierce BCA protein assay kit (Thermo Fisher Scientific, Inc.). The protein was separated by $15 \%$ SDS-PAGE and then transferred onto Immobilon nitrocellulose membranes (EMD Millipore). Subsequently, the membranes were blocked in $5 \%$ milk for $1 \mathrm{~h}$ at room temperature, and then incubated with the indicated primary antibodies $(1: 1,000)$ at $4{ }^{\circ} \mathrm{C}$ overnight. The antibodies were as follows: IGF-1 (cat. no. ab9572), Caspase-3 (cat. no. ab13847) and GAPDH (cat. no. ab181602) antibodies were purchased from Abcam. The membranes were then incubated with the secondary antibody goat anti-rabbit IgG H\&L (HRP) (cat. no. ab97051) for $1 \mathrm{~h}$ at room temperature. GAPDH served as a loading control and protein bands were quantified using ImageJ software 1.52a (National Institutes of Health).

$R N A$ isolation and reverse transcription-quantitative $(R T-q)$ $P C R$. Total RNA was isolated from tissues or cultured cells using TRIzol reagent (Thermo Fisher Scientific, Inc.) as described previously and RNA was reverse transcribed to complementary (c)DNA from $1 \mu \mathrm{g}$ total RNA by using AMV reverse transcriptase (Takara Bio Inc.) and a RT primer according to the manufacturer's protocol. The reaction conditions were as follows: $16^{\circ} \mathrm{C}$ for $30 \mathrm{~min}, 42^{\circ} \mathrm{C}$ for $30 \mathrm{~min}$ and $85^{\circ} \mathrm{C}$ for $5 \mathrm{~min}$. qPCR was performed by using a Taqman PCR kit on an Applied Biosystems 7300 sequence detection system (Applied Biosystems; Thermo Fisher Scientific, Inc.), with U6 as the internal control. Taqman probe of miR-155 (cat. no. A25576) and U6 (cat. no. 4427975) were purchased from Thermo Fisher Scientific, Inc. The reactions were performed in a 96 -well plate at $95^{\circ} \mathrm{C}$ for $10 \mathrm{~min}$, followed by 40 cycles of $95^{\circ} \mathrm{C}$ for $10 \mathrm{sec}$ and $60^{\circ} \mathrm{C}$ for $1 \mathrm{~min}$.

To measure the level of IGF-1 mRNA, RNA was reverse-transcribed to cDNA from $1 \mu \mathrm{g}$ total RNA using AMV reverse transcriptase and oligo dT (Takara Bio Inc.). The reaction conditions were as follows: $42^{\circ} \mathrm{C}$ for $60 \mathrm{~min}$ and $70^{\circ} \mathrm{C}$ for $10 \mathrm{~min}$. qPCR was performed using SYBR Premix Ex Taq (Takara Bio Inc.) and the corresponding primers (Nanjing Synthgene Medical Technology Co., Ltd) on an ABI 7300 sequence detection system (Applied Biosystems; Thermo Fisher Scientific, Inc.). The reactions were performed in a 96 -well plate at $95^{\circ} \mathrm{C}$ for $5 \mathrm{~min}$, followed by 40 cycles of $95^{\circ} \mathrm{C}$ for $30 \mathrm{sec}, 60^{\circ} \mathrm{C}$ for $30 \mathrm{sec}$ and $72^{\circ} \mathrm{C}$ for $30 \mathrm{sec}$. GAPDH served as the internal control. The PCR primers were used as follows: IGF-1 sense, 5'-CGTCACCGGGACATTGAG TATTAC-3' and anti-sense, 5'-AATGCATGGTTAAACCGA TGCAAG-3'; GAPDH sense, 5'-GATATTGTTGACATCAAT GAC-3' and anti-sense, 5'-TTGATTTTGGAGGGATCTCG-3'.

Overexpression and knockdown of miR-155. miR-155 mimics (forward, 5'-UUAAUGCUAAUCGUGAUAGGG GU-3' and reverse, 5'-CUCCUACAUAUUAGCAUUAAC A-3'); control mimics (forward, 5'-UUCUCCGAACGUGUC ACGU-3', and reverse, (5'-ACGUGACACGUUCGGAGA A-3'); anti-miR-155 (5'-ACCCCUAUCACGAUUAGCAUU AA-3') and anti-miR control (5'-ACGUGACACGUUCGG
AGAA-3') were purchased from Nanjing Synthgene Medical Technology Co., Ltd. Cells were seeded in 6-well plates at a density of $2 \times 10^{5}$ per well and transfected using Lipofectamine 2000 (Thermo Fisher Scientific, Inc.) with a concentration of $50 \mathrm{nM}$ mimics or inhibitors. on the following day according to the manufacturer's protocol. Total RNA and protein were extracted after transfection for 24 and $48 \mathrm{~h}$, respectively.

The prediction of IGF-1 as a direct target of miR-155: miRWalk (http://www.umm.uni-heidelberg. de/apps/zmf/mirwalk/) was used as a tool to predict the direct target of miR-155.

Luciferase reporter assay. The entire 3'-UTR of IGF-1 was inserted into a luciferase reporter plasmid (Nanjing Synthgene Medical Technology Co., Ltd.). To examine the binding specificity, the sequences that interacted with miR-155 were mutated and the mutant IGF-1 3'-UTR was inserted into an equivalent luciferase reporter plasmid. For the luciferase reporter assay, cells were seeded in 24-well plates and each well was transfected with $1 \mu \mathrm{g}$ luciferase reporter plasmid, $1 \mu \mathrm{g} \beta$-galactosidase plasmid (internal control) and $100 \mathrm{pmol}$ miR-155 mimics, control mimics, anti-miR-155 or anti-miR control using Lipofectamine 2000 (Thermo Fisher Scientific, Inc.). After $48 \mathrm{~h}$, luciferase signals were measured using a luciferase assay kit (cat. no. E1500) according to the manufacturer's protocol (Promega Corp.) and $\beta$-galactosidase activity was measured using a microplate reader (Thermo Fisher Scientific, Inc.) at $420 \mathrm{~nm}$, using a $\beta$-galactosidase Assay kit (cat. no. RG0036) according to the manufacturer's protocol (Beyotime Institute of Biotechnology). Luciferase activities were normalized to $\beta$-galactosidase activities for each well.

Cell viability assay. Cells were seeded in 96-well plates at a density of 5,000 cells per well. After incubation overnight, cells were transfected with miR-155 mimics, control mimics, anti-miR-155, anti-miR control, IGF-1 plasmid or control plasmid using Lipofectamine 2000 (Thermo Fisher Scientific, Inc.) according to the manufacturer's protocol. At 2 days after transfection, the cell viability was then determined using an MTT assay according to the manufacturer's protocol. The purple MTT-formazan crystals was dissolved by adding $200 \mu 1$ dimethyl sulfoxide (Amresco, LLC) to wells, and the absorbance was record at $490 \mathrm{~nm}$.

Cell apoptosis assay. Cells were seeded in 12-well plates and transfected with miR-155 mimics, control mimics, anti-miR-155, anti-miR control, IGF-1 plasmid or control plasmid using Lipofectamine 2000 (Thermo Fisher Scientific, Inc.) according to the manufacturer's protocol. After $48 \mathrm{~h}$ of transfection, the attached and floating cells were harvested and re-suspended in binding buffer (100 mM HEPES, pH 7.4, $100 \mathrm{mM} \mathrm{NaCl}$ and $25 \mathrm{mM} \mathrm{CaCl}_{2}$ ) after a wash with cold PBS. Subsequently, the cells were stained with Annexin $\mathrm{V}$-FITC/propidium iodide (PI) for $15 \mathrm{~min}$ at room temperature in the dark. Apoptosis was measured using a flow cytometer (FACScalibur; BD Biosciences) using an Annexin V-FITC/PI staining kit (BD Biosciences) according to the manufacturer's protocol. Data were analyzed using FlowJo 7.6 Software (FlowJo LLC). 
$\Delta \mathrm{G}=-24.8 \mathrm{kcal} / \mathrm{mol}$

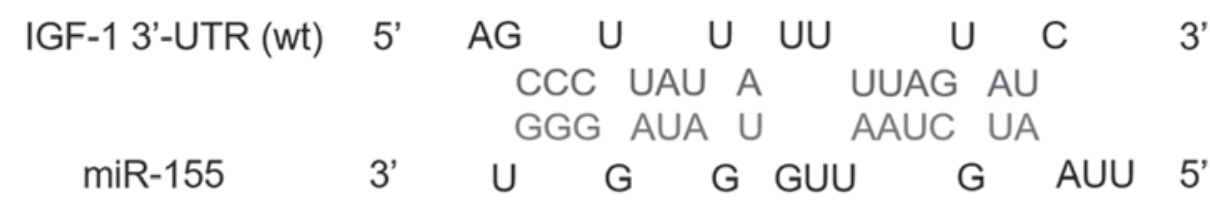

\section{IGF-13'-UTR (mut) 5' AGAUACGUCAUCUGAGUUGGC 3'}

Figure 1. Prediction of IGF-1 as a target of miR-155. Schematic illustration of the hypothetical duplexes formed through interactions between the binding site in the IGF-1 3'-UTR (top) and miR-155 (bottom). The sequence of the mutated IGF-1 3'-UTR and the predicted free energy are indicated. Grey letters indicate binding site interactions. IGF, insulin-like growth factor; UTR, untranslated region; miR, microRNA; wt, wild type; mut, mutant.

Animal experiment and histological analysis. Male BALB/c mice (age, 6 weeks) were purchased from the Model Animal Research Center of Nanjing University (Nanjing, China). The mice were maintained, in four mice per cage, at $21-23^{\circ} \mathrm{C}$, with a humidity of $40-60 \%$. The cage was maintained in a $12 \mathrm{~h}$ light dark cycle, with chow and water provided ad libitum. All animal protocols were approved by the Animal Care and Use Committee of Jiangsu Taizhou People's Hospital. Mice were administered streptozotocin $(40 \mathrm{mg} / \mathrm{kg}$ ) by a single tail-vein injection to induce diabetes (7). A blood glucose meter was used to measure the fasting blood glucose levels. After 3 days, those mice with blood glucose levels of $>16.7 \mathrm{mmol} / \mathrm{l}$ were selected as diabetic mice. The diabetic mice were treated with a control adenovirus, miR-155 adenovirus, IGF-1 adenovirus or miR-155 adenovirus plus IGF-1 adenovirus via tail vein injection. The amount of adenovirus was $10^{11}$ transforming units. $n=15$ in each group. After 6 weeks, mice were sacrificed and colonic smooth muscle tissues were dissected from the distal and proximal portion of the colon for further analysis. A total of three tissue samples of the same portion of the colonic smooth muscle tissues were subjected to H\&E staining by Nanjing Synthgene Medical Technology Co., Ltd. The IGF-1 and Caspase- 3 expression levels in the tissues were detected by western blot analysis.

Statistical analysis. All experiments were performed at least three times. HE staining was performed three times, and other experiments were performed six times, as indicating in the figure legends. Values are expressed as the mean \pm standard error of the mean. Statistical significance was determined using a Student's t-test or a one-way analysis of variance with post hoc Fisher's least significant difference test. $\mathrm{P}<0.05$ was considered to indicate a statistically significant difference.

\section{Results}

Prediction of IGF-1 as a target of miR-155. First, to identify a potential upstream regulator of IGF-1, miRWalk (21) was used to search miRNAs that may regulate IGF-1. miR-155, which was previously reported as a tumor-suppressive miRNA in colon cancer $(17,18)$ and a regulator of the inflammatory response in colonic mucosa (20), was then selected as the candidate miRNA that may target IGF-1 in colonic SMCs. Fig. 1 presents the potential binding between miR-155 and
IGF-1. The minimum free energy value of the hybridization was $-24.8 \mathrm{kcal} / \mathrm{mol}$, which is within the range of genuine miRNA-target interactions.

Validation of IGF-1 as a direct target of miR-155. Next, to validate whether IGF-1 is a direct target gene of miR-155, the expression IGF-1 was examined in mouse colonic SMCs after over-expression or knockdown of miR-155. Overexpression of miR-155 was achieved by transfection with miR-155 mimics and knock-down of miR-155 was realized through transfecting cells with anti-miR-155, which is a chemically synthesized anti-sense oligonucleotide designed to target mature miR-155. The over-expression and knockdown efficiencies were confirmed by measuring the expression levels of miR-155 in colonic SMCs by RT-qPCR. As presented in Fig. 2A, a $\sim 98$-fold increase in miR-155 levels was detected when cells were transfected with miR-155 mimics, while a $\sim 60 \%$ decrease in miR-155 levels was achieved when cells were transfected with anti-miR-155. The mRNA and protein levels of IGF-1 in these cells were then determined by RT-qPCR and western blot analysis, respectively. As indicated in Fig. 2B, the mRNA levels of IGF-1 remained consistent after over-expression or knockdown of miR-155. However, the protein levels of IGF-1 were significantly repressed upon overexpression of miR-155, whereas knockdown of miR-155 led to significantly increased IGF-1 protein levels $(\mathrm{P}<0.05$; Fig. $2 \mathrm{C}$ and $\mathrm{D})$. These results suggest that miR-155 negatively regulates IGF-1 expression at the post-transcriptional level.

To determine whether the negative regulatory function of miR-155 on IGF-1 was achieved through the binding of miR-155 to the predicted recognition site in the 3'-UTR of IGF-1 (Fig. 1), the full-length 3'-UTR of IGF-1 was inserted into the 3'-UTR of a firefly luciferase plasmid. The resulting plasmid was then transfected into colonic SMCs and miR-155 mimics or anti-miR-155 were co-transfected. Measurement of the luciferase activity in these groups of cells provided clues on whether miR-155 binds to the 3'-UTR of IGF-1. As presented in Fig. 3A, overexpression of miR-155 significantly reduced the luciferase signal, while knockdown of miR-155 significantly increased it $(\mathrm{P}<0.05)$. Furthermore, mutations were introduced into the binding site in the 3'-UTR of IGF-1 (Fig. 1) and this mutated 3'-UTR was inserted into the reporter vector downstream of the luciferase gene. The above experiments were re-performed with the mutant plasmid, revealing 

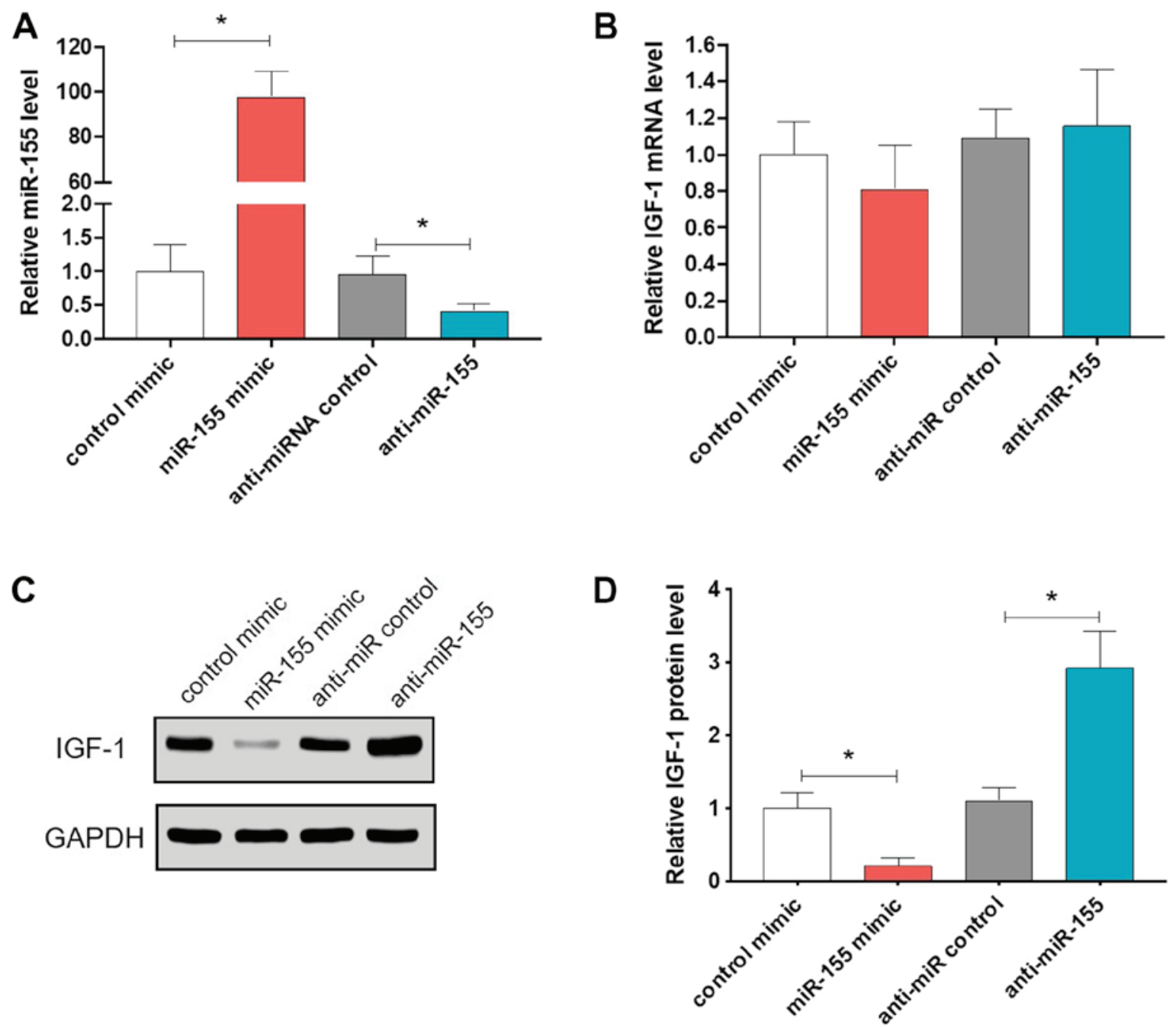

Figure 2. The effect of miR-155 on IGF-1 expression. Reverse transcription-quantitative PCR analysis of (A) miR-155 levels and (B) IGF-1 mRNA levels in colonic smooth muscle cells after transfection with control mimics, miR-155 mimics, anti-miR control or anti-miR-155. (C) Western blotting and (D) quantitative analysis of the IGF-1 protein levels in colonic smooth muscle cells after transfection with control mimics, miR-155 mimics, anti-miR control or anti-miR-155. The columns represent the mean \pm standard of the error of the mean $(n=6)$. "P<0.05, one-way analysis of variance with post hoc Fisher's least significant difference test. IGF, insulin-like growth factor; miR, microRNA.

that neither overexpression nor knockdown of miR-155 affected the luciferase signals (Fig. 3B). These results suggest that miR-155 directly binds to the 3'-UTR of IGF-1 to inhibit the translation of IGF-1 in colonic SMCs.

Role of miR-155 in the apoptosis of colonic SMCs. Since IGF-1 is able to inhibit the apoptosis of colonic SMCs (7), it was hypothesized that miR-155, which negatively regulates IGF-1, promotes the apoptosis of colonic SMCs through targeting IGF-1. To test this hypothesis, the viability and apoptosis of colonic SMCs were evaluated after overexpression or knockdown of miR-155. As expected, overexpression of miR-155 led to significantly decreased cell viability $(\mathrm{P}<0.05$; Fig. $4 \mathrm{~A})$ and increased apoptosis (Fig. 4B and C). By contrast, knockdown of miR-155 induced the opposite effects (Fig. 4A-C). Furthermore, to confirm that miR-155 promotes apoptosis via targeting IGF-1, an IGF-1 over-expression plasmid that expresses the full-length open reading frame of IGF-1 but without the 3'-UTR was constructed and co-transfected with miR-155 mimics into colonic SMCs. In comparison with cells transfected with control mimics or control plasmid, cells overexpressing IGF-1 exhibited significantly increased cell viability and significantly decreased apoptosis $(\mathrm{P}<0.05$; Fig. 4D-F), which is consistent with a previous study (7). However, co-transfection of miR-155 mimics with IGF-1 overexpression plasmid led to the reversal of the effect on cell viability and apoptotic rate (Fig. 4D-F). Taken together, these results indicate that miR-155 promotes apoptosis of colonic SMCs through targeting IGF-1.

Role of miR-155 in colonic dysmotility in diabetic mice. Given the evidence that IGF-1 can alleviate colonic dysmotility, it was then investigated whether miR-155 aggravates colonic dysmotility. Mice were first treated with streptozotocin to induce diabetes and colonic dysmotility according to a previous study (7). The expression levels of miR-155 in colonic muscle tissues of mice with or without colonic dysmotility were compared, revealing that miR-155 was significantly upregulated in colonic muscle tissues of mice with colonic dysmotility $(\mathrm{P}<0.001$; Fig. $5 \mathrm{E})$. To overexpress miR-155 or IGF-1, the corresponding adenovirus 

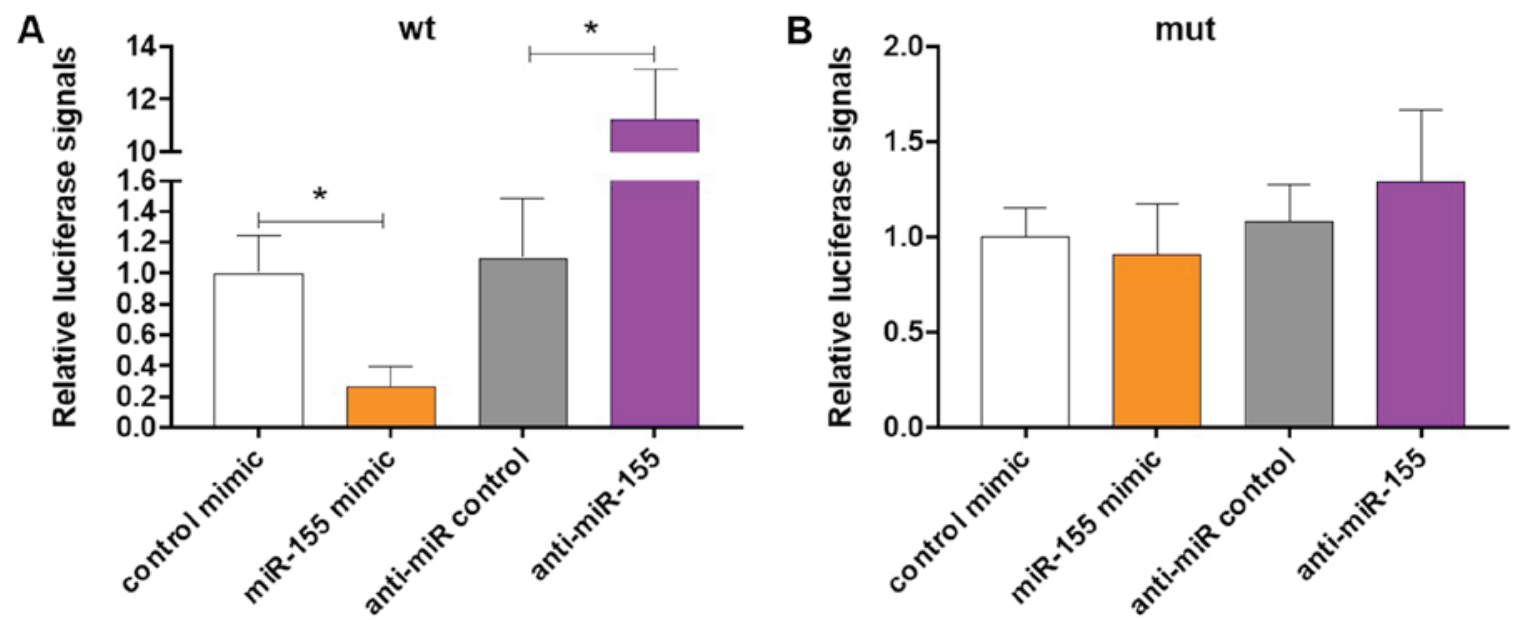

Figure 3. IGF-1 as a direct target of miR-155. Relative luciferase signals from colonic smooth muscle cells that were transfected with luciferase gene containing (A) wt or (B) mut miR-155 binding sites in the 3'-UTR of IGF-1 and co-transfected with control mimics, miR-155 mimics anti-miR control or anti-miR-155. The columns represent the mean \pm standard error of the mean $(\mathrm{n}=6) .{ }^{*} \mathrm{P}<0.05$, one-way analysis of variance with post hoc Fisher's least significant difference test. IGF, insulin-like growth factor; UTR, untranslated region; miR, microRNA; wt, wild-type; mut, mutant.
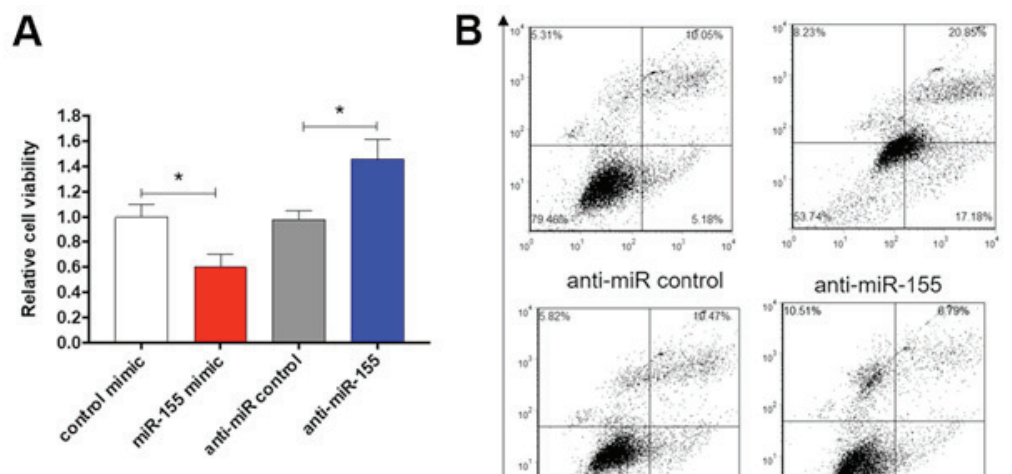

C
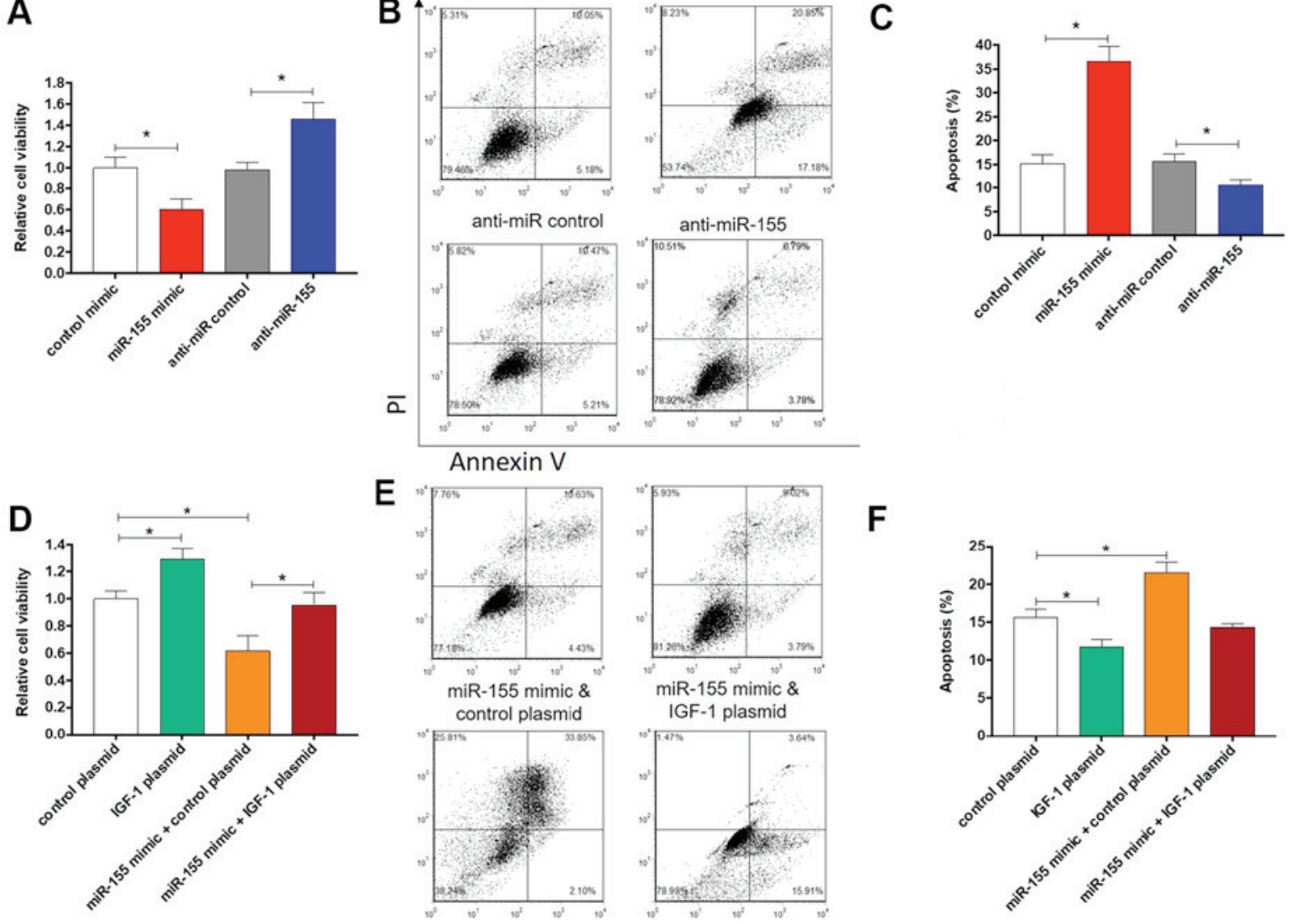

Figure 4. Effect of miR-155 and IGF-1 on colonic smooth muscle cells apoptosis. Relative cell viability and apoptotic rates of colonic smooth muscle cells that were transfected with (A-C) control mimics, miR-155 mimics, anti-miR control, anti-miR-155 or (D-F) control plasmid, IGF-1 plasmid, miR-155 mimics plus control plasmid or miR-155 mimics plus IGF-1 plasmid. (A and D) Cell viability. (B and E) Representative images and (C and F) quantitative analysis of apoptosis. The columns represent the mean \pm standard error of the mean $(n=6)$. ${ }^{*}<0.05$, one-way analysis of variance with post hoc Fisher's least significant difference test. IGF, insulin-like growth factor; UTR, untranslated region; miR, microRNA; PI, propidium iodide.

expression vectors were constructed. The diabetic mice were then treated with control adenovirus, miR-155 adenovirus, IGF-1 adenovirus or miR-155 adenovirus plus IGF-1 adenovirus via tail vein injection. The mice were sacrificed after 6 weeks and the colonic smooth muscle tissues were dissected, followed by H\&E staining, RT-qPCR analysis of miR-155 levels or western blot analysis of IGF-1 protein levels. The H\&E staining results are presented in Fig. 5A-D. In comparison with the control, the thickness of colonic smooth muscle tissues was increased in diabetic mice treated with IGF-1 adenovirus, which is consistent with the 
A

control adenovirus

B

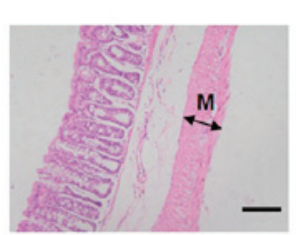

C

IGF-1 adenovirus

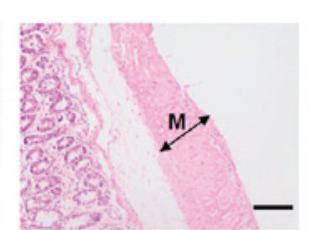

D miR-155 adenovirus

IGF-1 adenovirus

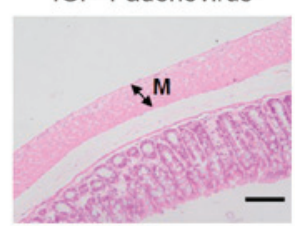

E

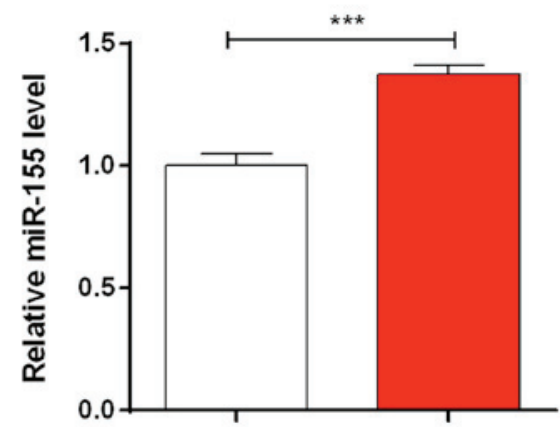

Figure 5. Effect of miR-155 and IGF-1 on the histopathological change of Colon tissues. Colon tissues from diabetic mice that were treated with (A) control adenovirus, (B) miR-155 adenovirus, (C) IGF-1 adenovirus or (D) miR-155 adenovirus plus IGF-1 adenovirus. The tissues were stained with hematoxylin and eosin (scale bar, $100 \mu \mathrm{M}$ ). (E) Reverse transcription-quantitative PCR analysis of miR-155 levels in the isolated colonic smooth tissues of normal mice and colonic dysmotility mice. The columns represent the mean \pm standard error of the mean $(n=6) .{ }^{* * * *} \mathrm{P}<0.0001$. M, muscle; IGF, insulin-like growth factor; miR, microRNA.

A
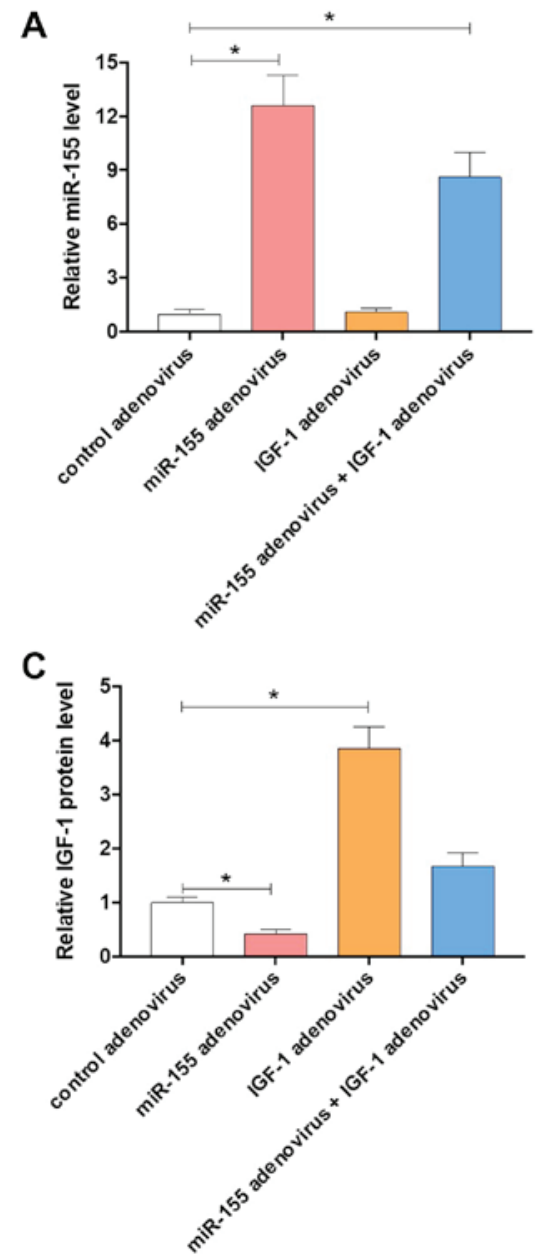

B

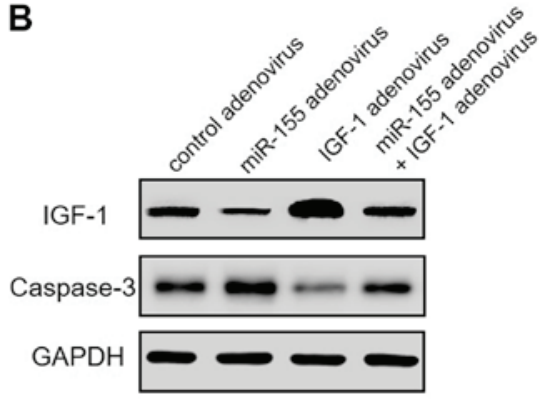

D

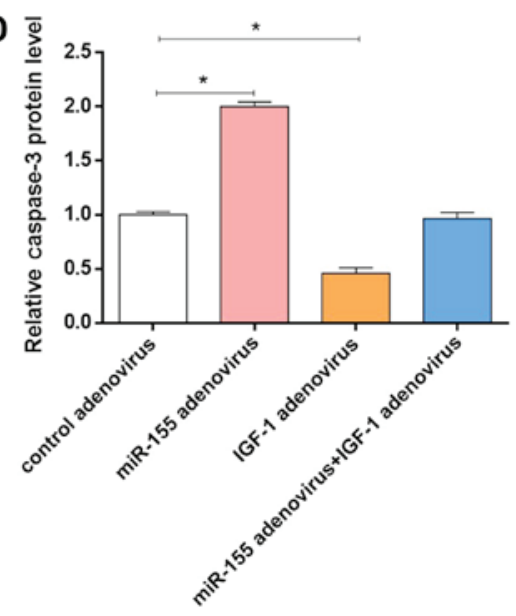

Figure 6. (A) miR-155 aggravated colonic dysmotility through targeting IGF-1. Reverse transcription-quantitative PCR analysis of miR-155 levels in the isolated colonic smooth muscle tissues. (B) Western blotting and (C) quantitative analysis of the IGF-1 and Caspase-3 protein levels in the isolated colonic smooth tissues. The columns represent the mean \pm standard error of the mean $(n=6) . ~{ }^{*} \mathrm{P}<0.05$, one-way analysis of variance with post hoc Fisher's least significant difference test. IGF, insulin-like growth factor; miR, microRNA. 
result of a previous study (7). By contrast, treatment of miR-155 adenovirus led to a decrease in the thickness of colonic smooth muscle tissues and co-treatment with IGF-1 adenovirus had rescuing effects (Fig. 5A-D). This result suggests that miR-155 promotes atrophy of colonic smooth muscle tissue in diabetic mice. To further validate this result, total RNA and protein in the isolated colonic smooth muscle tissues were extracted for RT-qPCR and western blot analysis. As presented in Fig. 6A, the levels of miR-155 in the colonic smooth muscle tissues of mice treated with miR-155 adenovirus were significantly increased $(\mathrm{P}<0.05)$. However, the protein levels of IGF-1 were decreased in the colonic smooth muscle tissues of mice treated with miR-155 adenovirus, but were upregulated in the colonic smooth muscle tissues of mice treated with IGF-1 adenovirus (Fig. 6B and C). The expression of Caspase-3 was also assessed (Fig. 6B), validating the function of miR-155 and IGF-1 in colonic dysmotility. Taken together, these results further demonstrate that miR-155 aggravates colonic dysmotility through targeting IGF-1.

\section{Discussion}

Gastrointestinal dysmotility is a complication of diabetes and is also accompanied with aging; it has become a serious threat to public health. Elucidation of the molecular mechanisms underlying gastrointestinal dysmotility is therefore urgently required. In general, gastrointestinal motility is provided by coordinated contractions of SMCs. Identification of the key molecules and associated molecular events of SMC contraction is thus essential, but the current knowledge remains insufficient. In a previous study, IGF-1 was reported to inhibit the apoptosis of colonic SMCs and alleviate colonic dysmotility (7), suggesting the important role of IGF-1 in colonic dysmotility. However, the upstream regulatory mechanisms of IGF-1 have remained elusive.

Certain miRNAs are considered to have key roles in gastrointestinal smooth muscle fibrosis and dysfunction. For instance, miR-143 and miR-145 were identified to be upregulated and involved in smooth muscle contractile dysfunction (22). miR-29b was reported to be downregulated and associated with gastrointestinal fibrosis (23). However, RNAs associated with colonic dysmotility as a complication of diabetes remains to be investigated in detail. In the present study, miR-155 was identified as an upstream regulator of IGF-1. The role of miR-155 in other diseases has been elucidated in considerable detail. For instance, miR-155 was previously demonstrated to induce functional impairment of vascular SMCs through downregulating soluble guanylyl cyclase (24). miR-155 was also reported to inhibit the proliferation of tumor cells via targeting cyclic AMP responsive element binding protein 1 (25) and to suppress the proliferation of fibroblasts during cardiac injury (26). The oncogenic functions of miR-155 in clear-cell renal cell carcinoma (27), hepatocellular carcinoma (28) and non-small cell lung cancer (29) have also been demonstrated. Among colonic diseases, miR-155 was identified as a tumor-suppressive miRNA in colon cancer $(17,18)$ and an important regulator of the inflammatory response in colonic mucosa (20). However, whether miR-155 participates in colonic dysmotility has remained elusive. In the present study, miR-155 was demonstrated to negatively regulate IGF-1 expression, which is achieved through direct binding of miR-155 to the
3'-UTR of IGF-1. It was also revealed that miR-155 targets IGF-1 to promote the apoptosis of colonic SMCs and aggravate colonic dysmotility. Taken together, the present results suggest a promoting effect of miR-155 in colonic dysmotility.

In conclusion, miR-155 was identified as a direct negative regulator of IGF-1, which promoted the apoptosis of colonic SMCs and aggravated colonic dysmotility in diabetic mice. These results may provide a novel therapeutic target that is miR-155 for the treatment of colonic dysmotility.

\section{Acknowledgements}

Not applicable.

\section{Funding}

The present study was supported by a grant from the National Natural Science Foundation of China (grant no. 81670490).

\section{Availability of data and materials}

The datasets used and/or analyzed during the present study are available from the corresponding author on reasonable request.

\section{Authors' contributions}

$\mathrm{XS}$ and $\mathrm{ZZ}$ performed the experiment. BY designed the research. XS and $\mathrm{ZZ}$ analyzed the data.XS and BY wrote the manuscript.

\section{Ethics approval and consent to participate}

All animal protocols were approved by The Animal Care and Use Committee of Jiangsu Taizhou People's Hospital.

\section{Patient consent for publication}

Not applicable.

\section{Competing interests}

The authors declare that they have no competing interests.

\section{References}

1. Nathan DM, Cleary PA, Backlund JY, Genuth SM, Lachin JM, Orchard TJ, Raskin P and Zinman B; DiabetesControl and Complications Trial/Epidemiology of Diabetes Interventions and Complications (DCCT/EDIC) Study Research Group: Intensive diabetes treatment and cardiovascular disease in patients with type 1 diabetes. N Engl J Med 353: 2643-2653, 2005.

2. Severino P, D'Amato A, Netti L, Pucci M, De Marchis M, Palmirotta R, Volterrani M, Mancone M and Fedele F: Diabetes mellitus and ischemic heart disease: The role of ion channels. Int J Mol Sci 19: E802, 2018.

3. Bytzer P, Talley NJ, Leemon M, Young LJ, Jones MP and Horowitz M: Prevalence of gastrointestinal symptoms associated with diabetes mellitus: A population-based survey of 15,000 adults. Arch Intern Med 161: 1989-1996, 2001.

4. Samsom M, Vermeijden JR, Smout AJ, Van Doorn E, Roelofs J, Van Dam PS, Martens EP, Eelkman-Rooda SJ and Van Berge-Henegouwen GP: Prevalence of delayed gastric emptying in diabetic patients and relationship to dyspeptic symptoms: A prospective study in unselected diabetic patients. Diabetes Care 26: 3116-3122, 2003. 
5. Rodrigues ML and Motta ME: Mechanisms and factors associated with gastrointestinal symptoms in patients with diabetes mellitus. J Pediatr (Rio J) 88: 17-24, 2012.

6. Sanders KM, Koh SD, Ro S and Ward SM: Regulation of gastrointestinal motility-insights from smooth muscle biology. Nat Rev Gastroenterol Hepatol 9: 633-645, 2012.

7. Sun M, Wang F and Feng P: Insulin-like growth factor-1 inhibits colonic smooth muscle cell apoptosis in diabetic rats with colonic dysmotility. Regul Pept 194-195: 41-48, 2014

8. He L and Hannon GJ: MicroRNAs: Small RNAs with a big role in gene regulation. Nat Rev Genet 5: 522-531, 2004.

9. Ambros V: The functions of animal microRNAs. Nature 431: 350-355, 2004.

10. John B, Enright AJ, Aravin A, Tuschl T, Sander C and Marks DS: Human MicroRNA targets. PLoS Biol 2: e363, 2004.

11. Bartel DP: MicroRNAs: Target recognition and regulatory functions. Cell 136: 215-233, 2009.

12. Friedman RC, Farh KK, Burge CB and Bartel DP: Most mammalian mRNAs are conserved targets of microRNAs. Genome Res 19: 92-105, 2009.

13. Lujambio A and Lowe SW: The microcosmos of cancer. Nature 482: 347-355, 2012.

14. Esquela-Kerscher A and Slack FJ: Oncomirs-microRNAs with a role in cancer. Nat Rev Cancer 6: 259-269, 2006.

15. Miao S, Mao X, Pei R, Song K, Lv Y, Jiang H, Li B, Yang X, Xiu C, Meng $\mathrm{H}$ and Sun J: MiR-448 inhibits laryngeal cancer metastasis by repressing AEG-1 expression. Int J Clin Exp Med 11: 1587-1596, 2018

16. Cao M, Bai L, Wang D, Zhai Q, Li Y, Hai J and Wang W: miRNA-33 expression and its mechanism in patients and model rats with type 2 diabetic nephropathy. Int J Clin Exp Med 11: 1661-1668, 2018.

17. Gao Y, Liu Z, Ding Z, Hou S, Li J and Jiang K: MicroRNA-155 increases colon cancer chemoresistance to cisplatin by targeting forkhead box O3. Oncol Lett 15: 4781-4788, 2018

18. Liu J, Chen Z, Xiang J, Gu X. MicroRNA-155 acts as a tumor suppressor in colorectal cancer by targeting CTHRC1 in vitro. Oncol Lett 15: 5561-5568, 2018.

19. Gao J, Zhao G, Li W, Zhang J, Che Y, Song M, Gao S, Zeng B and Wang Y: MiR-155 targets PTCH1 to mediate endothelia progenitor cell dysfunction caused by high glucose. Exp Cell Res 366: 55-62, 2018.
20. Pathak S, Grillo AR, Scarpa M, Brun P, D'Incà R, Nai L, Banerjee A, Cavallo D, Barzon L, Palù G, et al: MiR-155 modulates the inflammatory phenotype of intestinal myofibroblasts by targeting SOCS1 in ulcerative colitis. Exp Mol Med 47: e164, 2015.

21. Dweep H and Gretz N: miRWalk2.0: a comprehensive atlas of microRNA-target interactions. Nat Methods 12: 697, 2015.

22. Dahan D, Ekman M, Larsson-Callerfelt AK, Turczyńska K, Boettger T, Braun T, Swärd K and Albinsson S: Induction of angiotensin-converting enzyme after miR-143/145 deletion is critical for impaired smooth muscle contractility. Am J Physiol Cell Physiol 307: C1093-C1101, 2014.

23. Nijhuis A, Biancheri P, Lewis A, Bishop CL, Giuffrida P, Chan C, Feakins R, Poulsom R, Di Sabatino A, Corazza GR, et al: In Crohn's disease fibrosis-reduced expression of the miR-29 family enhances collagen expression in intestinal fibroblasts. Clin Sci (Lond) 127: 341-350, 2014.

24. Park M, Choi S, Kim S, Kim J, Lee DK, Park W, Kim T, Jung J, Hwang JY, Won MH, et al: NF-kB-responsive miR-155 induces functional impairment of vascular smooth muscle cells by downregulating soluble guanylyl cyclase. Exp Mol Med 51: 17, 2019.

25. Zhao XS, Han B, Zhao JX, Tao N and Dong CY: MiR-155-5p affects Wilms' tumor cell proliferation and apoptosis via targeting CREB1. Eur Rev Med Pharmacol Sci 23: 1030-1037, 2019.

26. Wang C, Zhang C, Liu L, A X, Chen B, Li Y and Du J: Macrophage-derived mir-155-containing exosomes suppress fibroblast proliferation and promote fibroblast inflammation during cardiac injury. Mol Ther 25: 192-204, 2017.

27. Ji H, Tian D, Zhang B, Zhang Y, Yan D and Wu S: Overexpression of miR-155 in clear-cell renal cell carcinoma and its oncogenic effect through targeting FOXO3a. Exp Ther Med 13: 2286-2292, 2017.

28. Li DP, Fan J, Wu YJ, Xie YF, Zha JM and Zhou XM: MiR-155 up-regulated by TGF- $\beta$ promotes epithelial-mesenchymal transition, invasion and metastasis of human hepatocellular carcinoma cells in vitro. Am J Transl Res 9: 2956-2965, 2017.

29. Liu F, Song D, Wu Y, Liu X, Zhu J and Tang Y: MiR-155 inhibits proliferation and invasion by directly targeting PDCD4 in non-small cell lung cancer. Thorac Cancer 8: 613-619, 2017. 